\title{
A assistência de enfermagem a partir da consulta remota: revisão de literatura
}

\author{
Nursing care based on remote consultation: a literature review
}

Atención de enfermería basada en la consulta remota: revisión de la literatura

Juliana de Souza Lima Coutinho ${ }^{1 *}$, Silvânia Medina de Souza ${ }^{1}$, Maria Angélica Alves de Macedo², Camila Santana Domingos ${ }^{1}$, Laylla Meireles de Souza ${ }^{1}$, Diego Dias Paz ${ }^{1}$, Luana Vieira Toledo.

\section{RESUMO}

Objetivo: Realizar uma Revisão de Literatura acerca da assistência de enfermagem a partir da consulta remota. Métodos: Foi realizada uma revisão integrativa da literatura em seis etapas, a partir do cruzamento dos descritores Enfermagem e "Consulta Remota" pela lógica booleana. A estratégia de busca foi realizada no portal de pesquisa Biblioteca Virtual em Saúde entre novembro e dezembro de 2020. Resultados: A combinação dos descritores resultou em 8 publicações, que após seleção e leitura na íntegra foram selecionados 4 para inclusão nesta revisão. Para a discussão dos artigos selecionados, foram elencadas duas categorias temáticas, sendo elas: A Teleconsulta de enfermagem, educação em saúde e a individualização do cuidado e A telesaúde e a aproximação das barreiras geográficas. Considerações finais: A Teleconsulta de enfermagem é uma pratica inovadora que pode auxiliar os pacientes no melhor gerenciamento de sua condição de patológica através da educação em saúde e empoderamento do sujeito para lidar com as adversidades da doença no ambiente domiciliar.

Palavras-chave: Enfermagem, Consulta remota, Processo de enfermagem.

\begin{abstract}
Objective: To carry out a literature review about nursing care from remote consultation. Methods: An integrative literature review was carried out in six steps, from the crossing of the descriptors Nursing and "Remote Consultation" using Boolean logic. The search strategy was performed on the Virtual Health Library search portal between November and December 2020. Results: The combination of descriptors resulted in 8 publications, which after selection and full reading, 4 were selected for inclusion in this review. For the discussion of the selected articles, two thematic categories were listed, namely: Nursing Teleconsultation, health education and the individualization of care and Telehealth and the approximation of geographical barriers. Final considerations: Nursing Teleconsultation is an innovative practice that can help patients to better manage their pathological condition through health education and the subject's empowerment to deal with the adversities of the disease in the home environment.
\end{abstract}

Key words: Nursing, Remote consultation, Nursing process.

\section{RESUMEN}

Objetivo: Realizar una Revisión de la Literatura sobre el cuidado de enfermería desde la consulta remota. Métodos: Se realizó una revisión integradora de la literatura en seis pasos, a partir del cruce de los descriptores Enfermería y "Consulta Remota" utilizando lógica booleana. La estrategia de búsqueda se realizó en el portal de búsqueda de la Biblioteca Virtual en Salud entre noviembre y diciembre de 2020. Resultados:

1 Universidade Federal de Viçosa (UFV), Viçosa - MG. *E-mail: jslcoutinho@gmail.com

2 Cooperativa de Trabalho Médico (Unimed-BH), Belo Horizonte - MG.

SUBMETIDO EM: 12/2021

ACEITO EM: 1/2022

PUBLICADO EM: 1/2022 
La combinación de descriptores resultó en 8 publicaciones, que luego de la selección y lectura completa, 4 fueron seleccionadas para su inclusión en esta revisión. Para la discusión de los artículos seleccionados se enumeraron dos categorías temáticas, a saber: Teleconsulta de enfermería, Educación en salud e individualización de la atención y Telesalud y aproximación de barreras geográficas. Consideraciones finales: La Teleconsulta de enfermería es una práctica innovadora que puede ayudar a los pacientes a manejar mejor su condición patológica a través de la educación para la salud y el empoderamiento del sujeto para enfrentar las adversidades de la enfermedad en el entorno del hogar.

Palabras clave: Enfermería, Consulta remota, Proceso de enfermería.

\section{INTRODUÇÃO}

Os avanços tecnológicos trouxeram importantes contribuições na prestação da assistência qualificada e segura pela incorporação de Tecnologias de Informação e Comunicação (TIC) na área da saúde, principalmente com o surgimento da internet e de tecnologias remotas (OLIVEIRA FM, 2021).

A superação das barreiras socioculturais, econômicas e geográficas a partir do uso destas tecnologias em práticas de saúde torna-se indispensável para formação, atualização e ampliação da prática profissional em saúde; além de contribuir com a melhoria do acesso aos cuidados de saúde, da qualidade do serviço prestado e da eficácia de diversas intervenções (OLIVEIRA FM, 2021; MINISTÉRIO DA SAÚDE, 2012).

Em 2007, foi instituído o Programa Nacional de Telessaúde para apoiar a Atenção Primária à Saúde. Em 2011 o programa foi reformulado e ampliado, passando a ser denominado Programa Nacional Telessaúde Brasil Redes. A partir disso Telessaúde passa a ser entendida como a utilização de TIC para atividades à distância relacionadas à saúde em seus diversos níveis (primário, secundário e terciário) (MINISTÉRIO DA SAÚDE, 2012).

A expressão Telessaúde é caracterizada como a prestação de assistência à saúde por meio do uso de TIC. Esse instrumento tem como base a telemática, a qual é definida como o estudo do emprego e manipulação da informação por meio do uso integrado de computador, seus acessórios e meios de comunicação (MAEYAMA MA e CALVO MCM, 2018).

Inicialmente a realização das ações de saúde a distância era definida como telemedicina, onde, por intermédio, do manejo da tecnologia de informação e telecomunicação eram realizadas a movimentação de dados médicos de diagnóstico, terapêutica e educação (OLIVEIRA FM, 2021). A ideia primordial é baseada no aprimoramento da acessibilidade do usuário ao serviço de saúde através da aplicação de diversos modelos de TIC, uma vez que essa ferramenta possibilita o acesso aos usuários independente de sua situação geográfica (BELBER GS, 2021).

No entanto, a palavra telemedicina promove um conceito restrito a área médica, excluindo, desse modo, os demais campos da saúde. Nesse sentido, o termo Telessaúde traz uma concepção mais ampla da utilização de TIC. Pois, a aplicabilidade desse recurso tem a nova finalidade, não só de viabilizar o trânsito de informações e serviços clínicos, administrativos e educacionais em saúde, mas também permite que todas as profissões da saúde possam manipular esse recurso (BELBER GS, 2021).

Na perspectiva de melhoria da assistência em saúde, os teleatendimentos se configuram como uma excelente estratégia, principalmente para as regiões carentes e remotas, onde é difícil o acesso a assistência especializada e presencial (NATIVIDADE TSS, et al., 2021).

Dentre as áreas que podem ser favorecidas pelo uso das TIC's, destaca-se a enfermagem. É bem estabelecido que as práticas de prevenção e promoção a saúde tem como subsídio a realização de educação em saúde e envolvimento do paciente como protagonista do seu processo saúde doença, sendo o enfermeiro um dos principais contribuintes dessa pratica (ALVES DS, et al., 2018). 
Nessa perspectiva, visando atender as demandas de saúde da atual conjuntura, a enfermagem emprega, de diversas formas, as TICs na sua prática profissional, seja por meio de sistemas que concedem acesso à informação em qualquer território geográfico, prontuários digitais e também fornecimento de atenção à saúde por meio de ligações telefônicas ou videoconferência (BARBOSA IA e SILVA MJP, 2017).

O enfermeiro corresponde a um papel impar nesse processo, sendo frequentemente responsável pelo acompanhamento tanto preventivo quando de monitoramento durante tratamentos, internações, pós operatórios ou altas hospitalares. Essas práticas podem ser potencializadas pelo acompanhamento remoto, tele monitoramento, tele consulta e outras modalidades de tele saúde (MARINHO OFP e ABRANCHES DC, 2018).

Nesse contexto, realizou-se este estudo com o objetivo de avaliar as publicações nacionais relacionadas a consulta de enfermagem remota.

\section{MÉTODOS}

Trata-se de uma Revisão Integrativa da Literatura. Esse tipo de estudo tem por objetivo levantar evidencias e informações sobre determinado tema a fim de se responder uma pergunta e aprofundar o conhecimento sobre determinado tema (SANTOS BATISTA L e KUMADA KMO, 2021; SOUSA, LMM, et al., 2018).

Buscou-se responder à seguinte questão norteadora: $O$ que se tem publicado na literatura nacional sobre a utilização da consulta remota na área de enfermagem?

Após definição do objetivo da pesquisa, foi realizada consulta ao Descritores em Ciências da Saúde (DeCS) a fim de elencar os descritores a serem utilizados na busca e os selecionados foram: "Enfermagem" e "consulta remota".

Para a realização da busca foi realizado o cruzamento dos descritores utilizando-se da lógica booleana no campo de busca. A estratégia utilizada na busca por descritores foi: Enfermagem AND "consulta remota". A busca na literatura foi realizada nos meses de novembro e dezembro de 2020, por meio do portal de pesquisa Biblioteca Virtual de Saúde (BVS) nas seguintes bases de dados: Literatura Latino-Americana e do Caribe em Ciências da Saúde (LILACS), Base de Dados de Enfermagem (BDENF), Medical Literature Analysis and Tetrieval System Online (MEDLINE) e Coleciona SUS.

Os critérios de inclusão foram: artigos científicos, escritos no idioma português, independente do ano de publicação, com isso esperou-se levantar o maior número de publicações já realizadas antes da data da busca a fim de compreender a temática desde sua origem e início das publicações. Foram excluídas as pesquisas no formato de editoriais, opiniões ou comentários, além de artigos que não abordaram o tema ou que não responderam à pergunta norteadora.

Após a realização da busca na literatura, foram aplicados os critérios de inclusão e exclusão a fim de selecionar os artigos que respondessem a pergunta de pesquisa.

Os estudos incluídos para discussão nesta revisão foram classificados quanto ao nível de evidência, sendo: Nível I, revisões sistemáticas ou metanálises de relevantes ensaios clínicos; nível II, evidências provenientes de pelo menos um ensaio clínico randomizado controlado e bem delineado; nível III, estudos controlados sem randomização; nível IV, estudos de coorte e de caso controle bem delineados; nível V, revisões sistemáticas de estudos descritivos e qualitativos; nível VI, evidências derivadas de um único estudo descritivo ou qualitativo; nível VII, opiniões de autoridades ou relatório de comitês de especialistas (MELNYK e FINEOUT-OVERHOLT, 2011).

Para análise e seleção das informações contidas em cada estudo selecionado, foi realizada a coleta de dados a partir de um instrumento de fichamento contendo: Título, autores, ano de publicação, tipo de estudo, objetivos do estudo, nível de evidencia e conclusão.

\section{RESULTADOS}

A combinação dos descritores resultou em 8 publicações, distribuídas nas 4 bases de dados conforme apresentado no Quadro 1. 
Quadro 1 - Resultado da busca eletrônica nas diferentes bases de dados científicas.

\begin{tabular}{|c|c|}
\hline Base de Dados & Número de Artigos \\
\hline BDENF & 3 \\
\hline MEDLINE & 2 \\
\hline LILACS & 2 \\
\hline Coleciona SUS & 1 \\
\hline Total & $\mathbf{8}$ \\
\hline
\end{tabular}

Fonte: Coutinho JSL, et al., 2021.

De acordo com a recuperação das publicações no período da busca, evidenciou-se a falta de estudos publicados na temática da Telessaúde na área de enfermagem, fato que pode ser justificado pela baixa adesão a essa modalidade de acompanhamento em saúde até o período da realização da busca.

Pela leitura dos títulos e resumos, foi possível excluir aqueles estudos que não abordaram o tema proposto nesta pesquisa. Dentre todos os estudos analisados, foram selecionados 4 artigos para leitura na íntegra, os quais todos responderam à questão norteadora e com isso constituíram a amostra final desta revisão (Figura 1).

Figura 1 - Processo de seleção amostral nas bases de dados nacionais e internacionais.

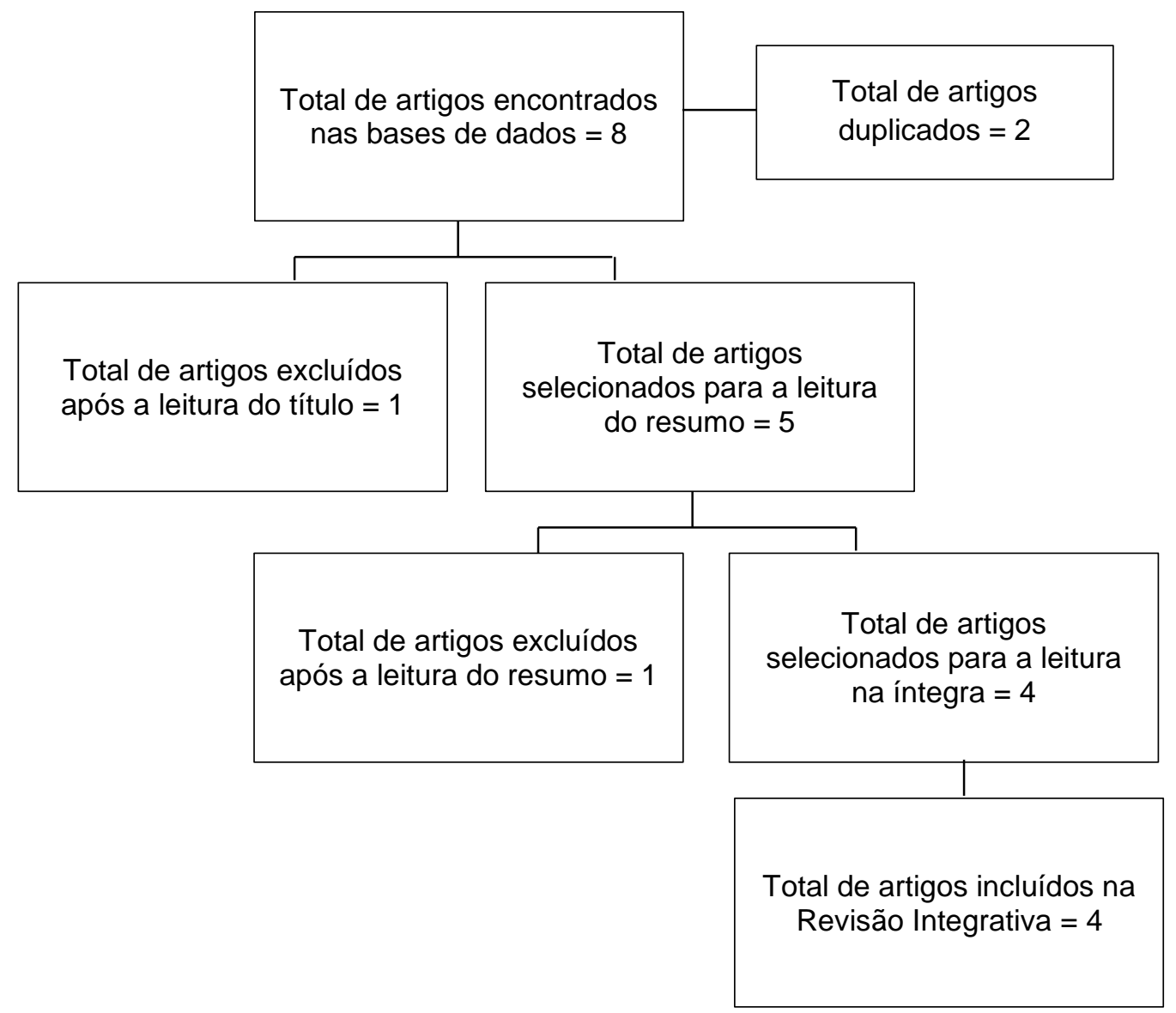

Fonte: Coutinho JSL, et al., 2021.

Para melhor compreensão dos estudos selecionados, foi elaborado um quadro para análise dos estudos com as seguintes variáveis: título, autor, ano, objetivo, Tipo de Estudo (TE), Nível de Evidência (NE) e conclusão (Quadro 2). 
Quadro 2 - Apresentação dos artigos segundo as variáveis eleitas.

\begin{tabular}{|c|c|c|c|c|}
\hline $\begin{array}{c}\text { Titulo } \\
\text { Autor, ano }\end{array}$ & Objetivo & TE & NE & Conclusão \\
\hline $\begin{array}{c}\text { Consulta de } \\
\text { enfermagem via } \\
\text { telefone: relatos dos } \\
\text { pacientes } \\
\text { submetidos à } \\
\text { quimioterapia } \\
\text { antineoplásica } \\
\text { (FERREIRA EB, et } \\
\text { al., 2014) }\end{array}$ & $\begin{array}{c}\text { Conhecer a } \\
\text { opinião dos } \\
\text { pacientes acerca } \\
\text { do } \\
\text { acompanhamento } \\
\text { telefônico } \\
\text { semanal } \\
\text { oferecido pela } \\
\text { equipe de } \\
\text { enfermagem }\end{array}$ & $\begin{array}{l}\text { Estudo } \\
\text { descritivo } \\
\text { longitudinal }\end{array}$ & VI & $\begin{array}{l}\text { O conhecimento das opiniões dos } \\
\text { participantes permitiu identificar } \\
\text { que a consulta de enfermagem } \\
\text { semanal via telefone possibilitou o } \\
\text { esclarecimento de dúvidas e } \\
\text { facilitou a recuperação do paciente } \\
\text { em domicílio, além de proporcionar } \\
\text { maior segurança para familiares e } \\
\text { cuidadores. }\end{array}$ \\
\hline $\begin{array}{l}\text { Efeito do } \\
\text { monitoramento } \\
\text { remoto de } \\
\text { enfermagem sobre o } \\
\text { excesso de peso de } \\
\text { mulheres: ensaio } \\
\text { clínico (PALMEIRA } \\
\text { CS, et al., 2019) }\end{array}$ & $\begin{array}{l}\text { Avaliar o efeito do } \\
\text { monitoramento } \\
\text { remoto de } \\
\text { enfermagem na } \\
\text { melhora das } \\
\text { medidas } \\
\text { antropométricas } \\
\text { de mulheres com } \\
\text { excesso de peso. }\end{array}$ & $\begin{array}{c}\text { Ensaio } \\
\text { clínico } \\
\text { randomizado }\end{array}$ & II & $\begin{array}{l}\text { O efeito do monitoramento remoto } \\
\text { foi benéfico na redução das } \\
\text { medidas antropométricas. }\end{array}$ \\
\hline $\begin{array}{c}\text { A construção } \\
\text { imaginativa de } \\
\text { cuidados: a } \\
\text { experiência de } \\
\text { profissionais de } \\
\text { enfermagem em um } \\
\text { serviço de } \\
\text { assistência remota } \\
\text { (ROMERO YMP, et } \\
\text { al., 2012) }\end{array}$ & $\begin{array}{l}\text { Compreender a } \\
\text { experiência de } \\
\text { interação dos } \\
\text { profissionais de } \\
\text { enfermagem com } \\
\text { os usuários }\end{array}$ & $\begin{array}{l}\text { Estudo } \\
\text { Transversal } \\
\text { descritivo }\end{array}$ & VI & $\begin{array}{c}\text { A compreensão teórica resultante } \\
\text { da análise interpretative permitiu a } \\
\text { construção do modelo teórico da } \\
\text { Construção Imaginativa do } \\
\text { Cuidado, que reflete os processos } \\
\text { interacionais que o profissional } \\
\text { vivencia quando presta cuidados } \\
\text { por meio do telefone. }\end{array}$ \\
\hline $\begin{array}{l}\text { Enfermagem em } \\
\text { estomaterapia no } \\
\text { Telessaúde uerj: } \\
\text { relato de experiência } \\
\text { (OLIVEIRA SOUZA } \\
\text { NVD, et al., 2014) }\end{array}$ & $\begin{array}{l}\text { Descrever um } \\
\text { relato da } \\
\text { experiência em } \\
\text { atividades } \\
\text { desenvolvidas } \\
\text { pelo Telessaúde } \\
\text { UERJ. }\end{array}$ & $\begin{array}{l}\text { Relato de } \\
\text { experiência }\end{array}$ & VI & $\begin{array}{c}\text { Esta atuação foi considerada } \\
\text { profícua, pois contribui para a } \\
\text { construção e a consolidação do } \\
\text { conhecimento técnico e científico } \\
\text { de profissionais da saúde, ajuda na } \\
\text { qualificação do cuidado e } \\
\text { possibilita o contato com } \\
\text { estratégias de ensino- } \\
\text { aprendizagem diferenciadas e } \\
\text { inovadoras. }\end{array}$ \\
\hline
\end{tabular}

Fonte: Coutinho JSL, et al., 2021.

Analisando os objetivos dos estudos selecionados, percebe-se que todos abordam como tema central alguma modalidade de tele saúde realizado por equipes de enfermagem.

Em relação ao idioma de publicação, todos os estudos foram publicados em português. Já no que tange aos anos de publicação, foram publicados entre 2012 e 2019, sendo 50\% publicados no ano de 2014 .

No que diz respeito ao nível de evidencia, $75 \%$ dos estudos selecionados foram classificados no nível IV evidências derivadas de um único estudo descritivo ou qualitativo, enquanto $25 \%$ dos estudos foram classificados no nível II evidências provenientes de pelo menos um ensaio clínico randomizado controlado e bem delineado. 


\section{DISCUSSÃO}

Após a análise crítica dos estudos selecionados, foram elencadas duas categorias para a discussão dos artigos, sendo elas: Teleconsulta de enfermagem, educação em saúde e a individualização do cuidado; Telessaúde e a aproximação das barreiras geográficas.

\section{Teleconsulta de enfermagem, educação em saúde e a individualização do cuidado}

As práticas de saúde baseadas no cuidado são o cerne da profissão enfermeiro, sendo a enfermagem caracterizada como a ciência do cuidar. Para sua pratica, o enfermeiro lança mão de ações que objetivam identificar os problemas de saúde do paciente, elaborar planos de cuidados que melhorem suas condições e definir intervenções especificar para este paciente. Para esse processo, se dá o nome de consulta de enfermagem (CONSELHO FEDERAL DE ENFERMAGEM - COFEN, 2019).

$\mathrm{Na}$ consulta de enfermagem é realizado o histórico de enfermagem, que se baseia na coleta de dados sobre o processo saúde-doença do paciente, além do levantamento de problemas e diagnósticos de enfermagem prioritários. Dessa forma, são identificadas as necessidades do paciente e as possíveis intervenções e orientações relacionadas a educação em saúde para sua prevenção, acompanhamento ou resolução de problemas identificados (BARRA DCC, et al., 2021).

A consulta de enfermagem de forma remota, também denominada de Teleconsulta de enfermagem, segue os mesmos princípios. Inicia-se a partir da coleta de dados e histórico de enfermagem, passa pela identificação dos problemas a serem trabalhados, define as intervenções para a resolução dos problemas, implementa a intervenção definida, geralmente por meio da educação em saúde e, posteriormente, avalia à adesão do paciente às orientações (CAETANO R, et al., 2020).

Outra estratégia que surge como adicional à Teleconsulta é o Telemonitoramento, caracterizado pelo acompanhamento telefônico dos pacientes de acordo com as necessidades individualizadas. $O$ Telemonitoramento tem como característica dar seguimento a um atendimento presencial prévio, por exemplo acompanhamento de pós-operatório, ou monitoramento de efeitos colaterais de terapias como rádio ou quimioterápicos (LOURENÇO GM, et al., 2021).

Tanto a Teleconsulta como o Telemonitoramento devem ser considerados espaços ricos para a troca de conhecimentos e adoção de práticas de educação em saúde, com vistas à resolução dos problemas identificados, pois permite uma aproximação do profissional junto ao paciente que, possivelmente, não seria realizada com tanta frequência no modelo de atendimento presencial (BARRA DCC, et al., 2021).

Em um estudo sobre Telemonitoramento de enfermagem em pacientes oncológicos pós quimioterápicos foi evidenciado o papel da educação em saúde nessa modalidade de atendimento. Identificou-se a promoção da autonomia e redução dos efeitos adversos das drogas diante do acompanhamento e educação em saúde. Os pacientes relataram que o acompanhamento telefônico com a enfermeira permitiu identificar e esclarecer se os sintomas que sentiam eram relacionados ou não ao uso de medicamentos ou complicações da doença (FERREIRA EB, et al., 2014).

Muitos pacientes que realizam terapias, cirurgias ou procedimentos hospitalares ou ambulatoriais apresentam diversas dúvidas quando chegam em casa. Dúvidas estas, que por diversos fatores não surgem durante o procedimento, tais como: ansiedade, medo do próprio procedimento, preocupação com deslocamento ou outras ocupações. Um estudo com Telemonitoramento na atenção primária à saúde, identificou que quando o paciente se encontra em casa e vai refletir sobre a terapêutica, é comum que ocorra questionamentos sobre o correto tratamento, os quais só serão respondidos na próxima consulta presencial. Nesse contexto, o Telemonitoramento pode ser visto como uma potencialidade para o esclarecimento das dúvidas e questionamentos em tempo oportuno, proporcionando maior segurança no seguimento de saúde (LOURENÇO GM, et al., 2021).

A percepção positiva por parte dos participantes em relação ao Telemonitoramento de enfermagem foi tão evidente em um dos estudos, que os participantes manifestaram desejo de que a prática do Telemonitoramento de enfermagem fosse disseminada e que todos os pacientes pudessem receber o mesmo acompanhamento e assistência de enfermagem. Esse desejo relaciona-se ao fato de considerarem o 
Telemonitoramento fundamental no manejo dos efeitos colaterais da terapia e no apoio emocional durante 0 tratamento (FERREIRA EB, et al., 2014).

A prática do Telemonitoramento é considerada inovadora e tem ganhado espaço, com potencial para ajudar pacientes portadores de condições crônicas ou agudas, potencializando sentimentos de empoderamento e auxiliando no melhor gerenciamento da doença. Além disso, apresenta como vantagens a maior criação de vínculo com os profissionais da equipe de saúde (PALMEIRA CS, et al., 2019; ROMERO YMP, et al., 2012).

No que concerne a aplicação de TIC's na assistência em saúde, Barbosa IA e Silva MJP (2017), em seu estudo, fazem uma reflexão a respeito das diferenças existentes entre os cenários presencial e virtual em referência as características interpessoais envolvidas no atendimento pelo Telessaúde. O contexto não presencial tende a descaracterizar as relações entre as pessoas que participam do processo, devido às barreiras sensoriais e a limitação da análise das expressões não verbais durante a consulta. Assim, o modo presencial proporciona ao profissional uma melhor investigação da demanda do paciente, dado que é possível avaliar questões que vão além do relato verbal.

Por outro lado, percebe-se que apesar da limitação em relação a análise das expressões não verbais, o Telessaúde apresenta mais pontos positivos do que negativos, destacando-se o fato de permitir que os pacientes e familiares permaneçam em casa, diminuindo o custo com o tratamento. Além disso, possibilita que o paciente seja acompanhado por diversas especialidades em um único local, facilitando a movimentação do paciente na rede de saúde (COSTA T, et al., 2021)

Visto isso, vale reforçar que essa modalidade de atendimento se tornou uma ferramenta excepcional para realização de monitoramento dos pacientes, esclarecimento de dúvidas e consultas com menor complexidade. Destaca-se a necessidade de criação de protocolos de atendimento que busquem aumentar a interação e reduzir a distância, proporcionando, desse modo, maior eficácia dessa forma de atendimento (BARBOSA IA e SILVA MJP, 2017).

Um ensaio clinico randomizado realizado com 101 mulheres em um serviço ambulatorial de referência para obesidade na Bahia avaliou o efeito do monitoramento remoto de enfermagem nas medidas antropométricas de mulheres com excesso de peso. Enquanto o grupo controle compareceu apenas às consultas de acompanhamento de rotina, o grupo intervenção, além dessas consultas, recebeu o monitoramento remoto constituído pelas ligações telefônicas. Ao final da intervenção os grupos foram reavaliados e percebeu-se que o monitoramento remoto apresentou efeito benéfico quanto à redução das medidas antropométricas das mulheres do grupo de intervenção quando comparadas ao grupo controle (PALMEIRA CS, et al., 2019).

Outro estudo analisou a Telerreabilitação de pacientes e identificou como uma boa ferramenta para que os profissionais possam manter continuidade da assistência. Nesse mesmo estudo concluiu-se que as facilidades de uso dos dispositivos móveis e acesso à internet fazem com que o Telemonitoramento seja acessível e com grande potencial de adesão do paciente (RUARO MB, 2022)

O paciente quando acompanhado pelo serviço de saúde tende a aplicar e seguir com maior rigidez as orientações dadas pela equipe, visto que está sendo acompanhado de "perto". Essa sensação pode ser considerada como uma cobrança positiva, pois estimula que o paciente se mantenha nos hábitos orientados pela equipe até o próximo contato (PALMEIRA CS, et al., 2019).

No Telemonitoramento o paciente experimenta a valorização e sentimento de importância. Isso aumenta a autoestima e tem efeito na sua postura frente ao enfrentamento de suas doenças. Nesse momento, também é notado um aumento de confiança na equipe, já que se percebe o interesse em acompanhamento e dar orientações continuar e monitoramento do estado do paciente (FERREIRA EB, et al., 2014; PALMEIRA CS, et al., 2019).

Um dos estudos identificou uma aprovação de $100 \%$ dos pacientes em relação a assistência de enfermagem por meio do acompanhamento telefônico. Essa rotina permite dar continuidade e reforçar a educação em saúde, além de avaliar a adesão do paciente ao tratamento e seus cuidados (FERREIRA EB, et al., 2014; PALMEIRA CS, et al., 2019; ROMERO YMP, et al., 2012). 
Essa pratica pode assim, diminuir o número de visitas de emergência, internações evitáveis e proporcionar melhor controle de sintomas físicos e emocionais entre o domicilio e hospital. Assim, temos o uso do telefone como uma importante ferramenta de comunicação-enfermeiro paciente (FERREIRA EB, et al., 2014).

\section{Telessaúde e a aproximação das barreiras geográficas}

As tecnologias de informação e comunicação em saúde (TIC'S) vem em uma crescente por seu potencial de alternativa para educação em saúde e abordagens a distância. Permite o oferecimento de cursos, aperfeiçoamento profissional e acesso ao paciente para utilização do serviço de saúde (OLIVEIRA SOUZA NVD, et al., 2014).

A utilização dos serviços de saúde, por vezes, é dificultada por questões relacionadas ao acesso físico de determinados serviços. Nesse contexto, a Telessaúde pode ser eficaz para abordar e/ou acompanhar o paciente de forma alternativa, com maior comodidade, em qualquer ambiente. Essa prática tem várias potencialidades como alcançar pacientes com barreiras geográficas, minimizar as barreiras financeiras devido a ausência de deslocamento e dificuldades de acesso, além de permitir maior flexibilidade de horários e alcançar grande número de pacientes com incompatibilidade de agenda (PALMEIRA CS, et al., 2019).

Uma das modalidades da Telessaúde é a "Teleconsultoria". Essa modalidade foi definida pelo Ministério da Saúde (2021) como uma consulta para discussão entre profissionais ou gerentes do serviço de saúde, realizada de forma sistematizada pelo registro da consultoria. Seu principal objetivo é sanar dúvidas sobre a prática clínica ou em relação a processos de trabalho (MINISTERIO DA SAÚDE, 2012).

Em um estudo onde as autoras descreveram a experiência na realização de Teleconsultorias de enfermagem foi evidenciado que essa prática permitiu a ampliação de conhecimentos através do recebimento on-line de questionamentos sobre os cuidados a pacientes portadores de estomas, feridas agudas e crônicas, identificada como responsável pela quebra da barreira geográfica. As solicitações de consultorias e esclarecimentos vieram direcionadas de diversas localidades do Brasil. Assim, a Teleconsultoria foi considerada também uma estratégia que ajuda a qualificar o cuidado prestado na área da enfermagem e contribui para a capacitação de outros profissionais em outras regiões, que não teriam acesso ao serviço de forma presencial (OLIVEIRA SOUZA NVD, et al., 2014).

Assim, considerando o que estudos têm levantado, a Telessaúde tem sido avaliada como importante ferramenta para o enfermeiro, pois pode exercer influência também na redução do tempo de atendimento e dos custos de deslocamento de pacientes e profissionais de saúde; na satisfação dos sujeitos envolvidos no cuidado prestado e no aperfeiçoamento do serviço prestado (BARBOSA IA e SILVA MJP, 2017).

Vale ressaltar que, apesar dos artigos terem sido produzidos em período anterior à pandemia da COVID19, verifica-se que no contexto pandêmico a temática tornou-se ainda mais evidente. A Telessaúde ocupa papel de destaque no atendimento e acompanhamento seguro dos pacientes, devido sua vantagem de reduzir a circulação dos usuários nos estabelecimentos de saúde e, consequentemente, o risco de propagação do vírus da COVID-19 (COSTA PEREIRA FA e CORREIA DMS, 2020).

Nesse contexto, o alcance da Telessaúde tem ampliado para o combate da pandemia pelo novo coronavírus (2019-nCoV ou SARS-CoV-2), com a finalidade de promover consultas, esclarecimentos, encaminhamentos e orientações aos pacientes pelo uso de meios de TIC's (COFEN 2020; COSTA PEREIRA FA e CORREIA DMS, 2020).

As consultas remotas ganharam assim, visibilidade com a pandemia, pois foram consideradas eficazes para o atendimento às necessidades de saúde da população ao mesmo tempo que reduziram a circulação de pessoas e, consequentemente, minimizaram o risco de propagação do vírus (SILVA RS, et al., 2021).

Dessa forma, sua utilização para promoção e educação em saúde, pode ser uma importante ferramenta para o enfrentamento da pandemia do novo coronavírus, além de contribuir para a sua aceitação em diversas áreas assistenciais em saúde (CAETANO R, et al., 2020). 


\section{CONSIDERAÇÕES FINAIS}

Nacionalmente, a produção científica sobre consulta remota de enfermagem é escassa. Ainda assim, evidencia-se seu grande potencial, sendo considerada uma pratica inovadora que pode auxiliar os pacientes no melhor gerenciamento de sua condição de patológica. A partir dessa tecnologia assistencial é possível desenvolver ações de educação em saúde, com vistas ao empoderamento do sujeito para lidar com as adversidades da doença no ambiente domiciliar. A limitação da utilização da Teleconsulta de enfermagem encontra-se na dificuldade de acesso a tecnologias, como telefone/aparelho celular e conectividade de rede telefônica e internet, por partes de alguns pacientes.

\section{REFERÊNCIAS}

1. ALVES DS, et al. Telessaúde como suporte na assistência da enfermagem em obstetrícia. Enfermagem Brasil, 2018; 17(5): 471-479.

2. BARBOSA IA, SILVA MJP. Nursing care by telehealth: what is the influence of distance on communication?. Rev. Bras. Enferm. [Internet]. 2017; 70(5):928-34.

3. BARRA DCC, et al. Validação de diagnósticos de enfermagem para consulta de enfermagem na visita domiciliar ao adulto. Revista Brasileira de Enfermagem, 2021; 74.

4. BELBER GS, et al. Contribuições do Programa Nacional Telessaúde Brasil Redes na formação de recursos humanos na atenção básica. Brazilian Journal of Development, 2021; 7(1): 1198-1219.

5. CAETANO R, et al. Desafios e oportunidades para Telessaúde em tempos da pandemia pela COVID-19: uma reflexão sobre os espaços e iniciativas no contexto brasileiro. Cadernos de Saúde Pública, 2020; 36: e00088920.

6. CONSELHO FEDERAL DE ENFERMAGEM (COFEN). Resolução Cofen № 606/2019. Brasília, DF: COFEN, 2019. Disponível em: http://www.cofen.gov.br/resolucao-cofen-no-606-2019_70088.html . Acessado em 16 de dezembro de 2021.

7. CONSELHO FEDERAL DE ENFERMAGEM (COFEN). Resolução Cofen № 634/2020. Brasília, DF: COFEN, 2020. Disponível em: http://www.cofen.gov.br/resolucao-cofen-no-0634-2020_78344.htm . Acessado em 16 de dezembro de 2021.

8. COSTA PEREIRA FA, CORREIA DMS. Uso da teleconsulta pelo enfermeiro a cardiopatas: uma reflexão durante pandemia por COVID-19 no Brasil. Enfermagem em Foco, 2020; 11(2): 167-171.

9. COSTA T, et al. Oportunidade para telessaúde em tempos de pandemia: uma revisão integrativa. Brazilian Journal of Development, 2021; 7(11): 106419-106432.

10. FERREIRA EB, et al. Consulta de enfermagem via telefone: relatos dos pacientes submetidos à quimioterapia antineoplásica. Revista de Enfermagem do Centro-Oeste Mineiro, 2014; 4(2): 1090-1099.

11. LOURENCCO GM, et al. A experiência de Telemonitoramento por equipes de saúde da família em uma Unidade Básica de Saúde: breve relato. JMPHC| Journal of Management \& Primary Health Care, 2021; 13: e019-e019.

12. MAEYAMA MA, CALVO MCM. A integração do telessaúde nas Centrais de Regulação: a Teleconsultoria como Mediadora entre a Atenção Básica e a Atenção Especializada. Revista Brasileira de Educação Médica, 2018; 42: 6372.

13. MARINHO OFP, ABRANCHES DC. Uso de aplicativos por enfermeiros nas ações de prevenção em saúde. Revista Pindorama, 2018; 8(8): 7-7.

14. MELNYK BM, FINEOUT-OVERHOLT E. Prática baseada em evidências em enfermagem \& saúde: Um guia para as melhores práticas. $1^{\circ}$ ed. Lippincott Williams \& Wilkins, 2011; 3-24p.

15. MINISTÉRIO DA SAÚDE. Manual de Telessaúde para Atenção Básica / Atenção Primária à Saúde / Ministério da Saúde, Universidade Federal do Rio Grande do Sul. $2014 . \quad$ Disponível em: https://bvsms.saude.gov.br/bvs/publicacoes/manual_telessaude_atencao_basica.pdf. Acessado em: 16 de dezembro de 2021.

16. NATIVIDADE TSS, et al. Avaliação da satisfação e resolubilidade da plataforma Telessaúde redes no estado do Pará, Brasil. Revista Brasileira de Medicina de Família e Comunidade, 2021; 16(43): e2411.

17. NILSON LG, et al. Telessaúde: da implantação ao entendimento como tecnologia social. Revista Brasileira de Tecnologias Sociais, 2018; 5(1): 33-47.

18. OLIVEIRA FM, et al. Telemedicina e telecirurgia: uma prática possível. Múltiplos Acessos,2021; 6(3): 46-59.

19. OLIVEIRA SOUZA NVD, et al. Enfermagem em estomaterapia no Telessaúde UERJ: relato de experiência. Jornal Brasileiro de Telessaúde, 2014; 3(2): 70-72.

20. PALMEIRA CS, et al. Effect of remote nursing monitoring on overweight in women: clinical trial. Rev. Latino-Am. Enfermagem. Revista Latino-Americana de Enfermagem, 2019; 27:e3129.

21. ROMERO YMP, et al. A construção imaginativa de cuidados: a experiência de profissionais de enfermagem em um serviço de assistência remota. Revista Latino-Americana de Enfermagem, 2012; 20: 693-700.

22. RUARO MB, et al. Prospecção de Tecnologias para telerreabilitação: inovação nos atendimentos fisioterapêuticos. Cadernos de Prospecção, 2022; 15(1): 161-177.

23. SANTOS BATISTA L, KUMADA KMO. Análise metodológica sobre as diferentes configurações da pesquisa bibliográfica. Revista brasileira de iniciação científica, 2021; 8: e021029-e021029.

24. SILVA RS, et al. O Papel da telessaúde na Pandemia Covid-19: Uma Experiência Brasileira. Ciência \& Saúde Coletiva, 2021; 26: 2149-2157.

25. SOUSA, LMM, et al. Revisões da literatura: tipos, métodos e aplicações em enfermagem. Revista Portuguesa de Enfermagem de Reabilitação, 2018; 1(1): 45-54. 